\title{
Implication of Public Health Genomics for Low and Middle Income Countries
}

\author{
Neupane DC, \\ MPH, University of Southern Denmark. \\ Khanal V, MPH, Curtin University, Australia \\ CCorrespondence :Dinesh Neupane \\ Universtiy of Southern Denmark. \\ neupane.dinesh@gmail.com
}

\section{Introduction}

Genome is the sum total of genetic information of an individual, which is encoded in the structure of deoxyribonucleic acid (DNA). The study of the genome is termed "genomics"(WHO, 2002) and the application of genomics to population level is called public health genomics. The use of knowledge from the advancement in genomes holds considerable potential for the development of new health care innovations in the coming decades. (WHO, 2002). World Health Organization Report of the Advisory Committee on Health Research believed that in the long run the information generated by genomics will have major benefits for the prevention, diagnosis and management of many diseases which hitherto have been difficult or impossible to control. Pang claimed that the current revolution in genomics, culminating in the sequencing of the human genome has tremendous potential to improve health globally (Pang, 2002). These include communicable and genetic diseases, together with other common killers or causes of chronic ill health, including cardiovascular disease, cancer, diabetes, the major psychoses, dementia, rheumatic disease, asthma, and many others. On the other hand, genetic modification of crops has also great potential for improving human health through nutritional gains and the production and delivery of vaccines and therapeutic agents (WHO, 2002).

\section{Application to Low and Middle Income Countries}

Genomics hold great potential to fight diseases that disproportionately affect the world's poorest people (Daar, Berndtson, Persad, \& Singer, 2007). The advancement in public health genomics can alleviate both infectious diseases and non-infectious chronic diseases in low and middle income countries. However, ensuring that advances in genomics are applied to the health improvement of people living in such countries is an important contemporary challenge (Pang, 2003). A key issue in the post genomics era will be who will pay to test, develop, and deliver important vaccines, drugs, and diagnostic procedures for diseases of the low and middle income countries, and who will ensure equitable access to those who need it most (Pang, 2003).

Drugs and interventions derived from genomics are likely to be expensive, and of particular interest is how these advances will affect the health of people living in the low and middle income countries (Pang, 2002). The reality that much of the advances in genomics were made, and in part are owned, by the developed world has given rise to the concern that a genomics divide will be created that will further widen the equity gap in health between rich and poor nations(Singer P, 2001).According a report released by the World Health Organization in 2002 points out that approximately $80 \%$ of investments in genomics in 2000 were made in the United States, and $80 \%$ of the DNA patents in genomics in the period 1980 through 1993 were held by US companies (WHO, 2002). The lack of biotechnology and information technology development in many low and middle income countries is also of concern (WHO, 2002). Yet, the bulk of genomics research is not directed at these diseases, owing to the brutal reality that research is largely driven by market forces-its products are probably unaffordable to the poor living in the developing countries(Pang, 2003). There is still an ongoing debate regarding the genomics and its application in low and middle income countries. Some believe that genomics has diverted previous resources away from the core issues of global public health such as provision of clean water, safe food, proper sewage disposal, descent housing and access to maternal and child care (Garrett, 2001). Some of the claims for the medical benefits of genomics have undoubtedly been exaggerated, particularly with respect to the timescales required for them to come to fruition (WHO, 2002).

Despite having a tremendous potential to improve health status, very little effort has been made to address the public health problems via public health genomics in low and middle income countries. It is a true fact that most of the multinational organizations are not showing attention on this field because the most of the people living in low and middle income countries are poor and they could not afford the high cost. On the other hand, many ongoing genomics researches in low and middle income countries are mainly due to easy set up and low ethical consideration requirement. However, there are only limited to finding a 
treatment mechanism and applying in the industrialized world. So, that the people of low and middle income countries are far behind getting benefit of it.

Public health genomics should not be viewed as a separate and alternative discipline of public health sciences. The criticism of genomics arguing diverting resources from current approach will be address only when public health genomics is viewed as a supplemental component of public health science. Efforts need to enhance to make the public health genomics more accessible, affordable and applicable to the people who are at the most need.

\section{Conclusion}

Genomics brings with it complex new ethical, legal, social, and economic implications as well as concerns about risks and hazards. Despite the positive impact, it also raised concerned about ethics, challenges and constrains which needs to be addressed effectively (Fukuyama, 2002). The ethical approval committees in low and middle income countries are relatively weak and they do not have enough skilled human resources who can distinguish potential benefit and impact of such studies. This can be made possible by involving all low, middle and high income countries. Strong international leadership by the scientific community, international organizations, governments, and industry is required through promotion of innovative partnerships and cooperation strategies in order to get the fruits of public health genomics by developing countries(Pang, 2002). The local institutional review board or research ethics committees in developing countries should be strengthened in order to protect the right of participants. A more acceptable approach would be to truly involve and empower scientists and institutions in developing countries, working together in equal partnership. To ensure that benefits are shared by developing countries, attention must be paid to complex ethical, legal, social, and economic issues, as well as to public education and engagement. Creative and equitable international mechanisms and goodwill are needed to turn high hopes into reality and allow the use of genomics to reduce health inequities between rich and poor nations(Pang, 2002).

\section{References}

1. Cardon, L., \& Bell, J. (2001). Associaiton study designs for complex diseases. Nat Rev Genet, 2(2), 91-99.

2. Donnelly, P. (2008). Progress and challenges in genome-wide association studies in humans. Nature, 456(7223), 728-731.

3. Daar, A. S., Berndtson, K., Persad, D. L., \& Singer, P. A. (2007). How can developing countries harness biotechnology to improve health? BMC Public Health, 7(346).

4. Fukuyama, F. (2002). Our Posthuman Future: Consequences of the Biotechnology Revolution. New
York: Farrar Strauss \& Giroux.

5. Garrett, L. (2001). Betrayal of Trust-The Collapse of Global Public Health. New York: Hyperion.

6. Hoedemaekers, R., Gordijn, B., Hekster, Y., \& Van, F. A. (2006). The complexities of ethical evlauaiton of genomics research. HEF Forum, 18(1), 18-36.

7. Killen, J., Grady, C., Folkers, G., \& Fauci,A. (2002). Ethics of clinical research in developing world. Nature Rev Immunol, 2, 210-215.

8. Molyneux, C., Peshu, N., \& Marsh, K. (2004). Understanding of informed consent in a low-income setting: three case studies from the Kenyan coast. Social Science \& Medicine, 59(12), 2547-2559.

9. Pang, T. (2002). The Impact of Genomics on Global Health. Am J Public Health, 92(7), 1077-1079.

10. Pang, T. (2003). Equal partnership to ensure that developing countries benefit from genomics. Nature genetics, 33, 18.

11. Singer P, D. (2001). Harnessing genomics and biotechnology to improve global health equity. Science, 294, 87-89.

12. Vries, J. d., Bull, S. J., Doumbo, O., Ibrahim, M., Mercereau-Puijalon, O., Kwiatkowski, D., et al. (2011). Ethical issues in human genomics research in developing countries. [Debate]. BMC Medical Ethics, 12(5).

13. WHO. (2002). Report of the Advisory Committee on Health Research. Geneva: World Health Organization.

14. WMA. (2008). Declaration of Helskinki: Ethical Principles for Medical Research Involving Human Subjects.

15. Zulfiqar, B. A. (2002). Ethics in international health research: a perspective from the developing world. Bulletin of the World Health Organization, 80(2), 114120. 